\title{
Nurse-led rapid-access chest pain clinics: effective, safe and here to stay
}

\author{
Authors: Richard Elis Knowles Jones, Katherine Groom, Carmen Zerafa, Paraic Cliffe, Pat Phen and Rajesh Aggarwal
}

\begin{abstract}
Aims
To analyse test utilisation and revascularisation rates in a large, nurse-led, rapid-access chest pain (RACP) clinic. In addition, to assess for subsequent acute coronary syndrome (ACS) presentations in patients referred with suspected stable coronary artery disease (SCAD).
\end{abstract}

\section{Methods}

Retrospective analysis of the 4,661 patients seen at a nurse-led RACP clinic between November 2011 and October 2015.

\section{Results}

2,477 non-invasive investigations were performed as the initial test to assess for SCAD. These resulted in 93 (4\%) revascularisation procedures. In contrast, 686 invasive coronary angiograms were performed as the initial investigation. These resulted in 261 (38\%) patients being revascularised. 59 (1.3\%) patients were admitted with an ACS requiring invasive management up to 365 days following their RACP appointment. Nine $(0.2 \%)$ patients had been discharged without any SCAD work up, $30(0.6 \%)$ were awaiting their index investigation, eight $(0.2 \%)$ presented with an ACS after a negative non-invasive test, and $12(0.3 \%)$ were awaiting revascularisation. SCAD was diagnosed in 933 (20\%) of our population.

\section{Conclusions}

Invasive coronary angiography remains a common initial investigation in patients being worked up for SCAD. In support of this strategy, a high proportion of these patients subsequently undergo revascularisation. Non-invasive testing is reserved for patients with a lower pre-test probability of SCAD and, as such, considerably fewer patients in these groups are revascularised. Reassuringly, patients with suspected SCAD have a low incidence of ACS up to 1 year following diagnosis. In conclusion, cardiac specialist nurses are skilled at detecting patients with SCAD and can deliver a safe and effective service.

Authors: Basildon and Thurrock University Hospitals NHS Foundation

Trust, Basildon, Essex, UK
Readdressing the use of non-invasive testing in lower-risk groups may be appropriate, as only a small proportion of these patients had obstructive coronary artery disease in our study.

\section{Conflict of interest statement}

No conflicts of interest identified. 